\title{
Intention, image et singularité selon le premier Sartre : Réflexions sur les propriétés et les fonctions de l'acte d'imagination
}

\author{
Par FABIO RECCHIA \\ Université de Liège
}

Résumé Partant d'une problématisation de la méthode phénoménologique mise en jeu par L'Imaginaire, nous proposons de relire les développements de ce texte à partir de la perspective offerte par la théorie de l'intentionnalité $\mathrm{du}$ premier Sartre. Nous tenterons ainsi de dégager l'unité systématique du projet sartrien d'une "phénoménologie de l'image » en le rapportant aux propositions philosophiques posées de manière décisive par «l'article sur l'intentionnalité ». Par ce biais, il s'agira également de fixer les propriétés de la conscience imageante, ainsi que les fonctions jouées par l'acte d'imagination dans l'économie générale de la conscience, en particulier dans l'événement de sa personnalisation. De sorte que nous parcourons les tensions reliant l'imagination de la conscience à son ipséité ; tandis que, du point de vue de l'histoire de la philosophie, nous examinerons certaines des torsions qu'appliqua Sartre sur l'analyse husserlienne de l'image afin d'approfondir celle-ci dans la direction d'une philosophie de la liberté personnelle du sujet.

\section{Introduction}

L'Imaginaire de Sartre «a pour but de décrire la grande fonction "irréalisante" de la conscience ou "imagination" et son corrélatif noématique, l'imaginaire ${ }^{1}$. Quant au projet plus général de ce texte, il s'agit de «tenter

${ }^{1}$ J.-P. Sartre, L'Imaginaire, Paris, Gallimard, 2005, p. 13. 
une "phénoménologie" de l'image ${ }^{1}{ }^{1}$ consistant à pratiquer sur cette dernière une réflexion qui en dégage un contenu immédiatement certain : «l'essence de l'image $»^{2}$. Bien avant Sartre, Husserl avait formulé dans ses Recherches logiques l'idée originale d'une telle phénoménologie. En réaction à la Bildertheorie de Twardowski et ses embarrassantes régressions à l'infini, celui-ci proposait en effet une analyse de l'image qui, passant par une «attitude réflexive ${ }^{3}$ rivée aux essences, en délivre la structure intentionnelle. Or, on sait par ailleurs que cette posture réflexive est la source de la plupart des difficultés connues par la description phénoménologique. C'est que, comme le remarque l'auteur de L'Être et le Néant, évidemment à charge contre Husserl, « le regard réflexif altère le fait de conscience sur lequel il se dirige $»^{4}$.

Cela étant, bien au contraire des analyses précautionneusement formulées par l'œuvre husserlienne concernant le bon usage de la réflexion, L'Imaginaire semble, à l'inverse de l'ontologie phénoménologique, faire peu de cas des difficultés liées à la posture réflexive du phénoménologue. Sartre argue même que la réflexion est certaine en raison d'une vérité «qu'on sait depuis Descartes ${ }^{5}$, sous le haut patronage duquel, avec bonheur, on peut conduire sans encombre une description phénoménologique de l'image. « Une conscience réflexive », indique en ce sens la deuxième page de L'Imaginaire, «nous livre des données absolument certaines; l'homme qui, dans un acte de réflexion, prend conscience "d'avoir une image" ne saurait se tromper $»^{6}$.

Une telle affirmation réclame cependant d'être problématisée. Manifestement, Sartre valide la méthode de son analyse phénoménologique à partir de ce qu'il présente comme un acquis de l'histoire des idées de la philosophie française. Mais, dans ces conditions, L'Imaginaire ne présume-til pas acquise la légitimité de sa méthode? En conséquence, le texte ne manque-t-il pas de justifier la continuité logique entre la méthode et ses principaux résultats, et sans laquelle le lecteur de L'Imaginaire ne peut jamais être certain que la réflexion pratiquée par ce texte décrit l'acte d'imagination conformément à son intention? Bien plus, si L'Imaginaire ne fixe

\footnotetext{
${ }^{1}$ J.-P. Sartre, L'Imaginaire, op. cit., p. 17.

2 Ibid., p. 16.

${ }^{3}$ E. Husserl, Recherches logiques, Introduction, $\S 7$, trad. fr. H. Elie et alii, Paris, PUF, 2003, p. 11.

${ }^{4}$ J.-P. Sartre, L'Être et le Néant, Paris, Gallimard, 1976, p. 110.

${ }^{5}$ J.-P. Sartre, L'Imaginaire, op. cit., p. 15.

${ }^{6} \mathrm{Id}$.
} 
pas de manière rigoureuse les règles et les conditions d'application de l'attitude phénoménologique, Sartre ne développe-t-il pas, en définitive, une analyse de l'image qui n'a de phénoménologique que le label ? Selon nous, ce hiatus implique de réitérer à l'égard de L'Imaginaire le même soupçon que Sartre formulait à propos de son Esquisse d'une théorie des émotions: dans ce projet d'une phénoménologie de l'image, en effet, la « théorie est montrée mais non point démontrée $»^{1}$.

Cependant, loin de déclarer nulle et non avenue l'entreprise projetée par L'Imaginaire, nous voudrions considérer les difficultés sur lesquelles elle repose comme une invitation à relire les descriptions sartriennes de l'imagination. Dans ce texte, nous souhaitons en effet réexaminer ces développements à partir de l'angle de vue offert par l'œuvre du premier Sartre. De manière plus précise, le programme de L'Imaginaire consistant à analyser les propriétés et les fonctions de l'acte d'imagination, notre stratégie de lecture visera à réinscrire les descriptions de ce texte dans une perspective plus générale: celle de la théorie sartrienne de l'intentionnalité. Sur cette base, nous identifierons une continuité, non entre la méthode de L'Imaginaire et ses principaux résultats, mais entre ces derniers et certains principes fondamentaux de la philosophie sartrienne. D'où il suit que l'intentionnalité imaginative sera, dans notre texte, l'objet phénoménologique dont nous tenterons de dégager les caractéristiques spécifiques, et par lequel nous entreprendrons la légitimation des analyses composant la phénoménologie sartrienne de l'image. Nous examinerons, pour ce faire, l'extension chez Sartre des concepts d'intention, d'image et de singularité. Cela en focalisant principalement notre analyse sur quatre segments de son œuvre : «l'article sur l'intentionnalité $»^{2}$ chez Husserl, la première partie ainsi que la conclusion de L'Imaginaire, et la théorie du « circuit de l'ipséité » dans L'Etre et le Néant que nous relierons aux développements de L'Idiot de la famille sur la personnalisation de Flaubert.

Plus précisément, nous diviserons notre propos en quatre volets d'analyse. Nous montrerons tout d'abord que la théorie sartrienne de l'imagination peut être lue comme une déduction de prémisses posées en amont par « l'article sur l'intentionnalité » (1). Ensuite, nous examinerons les fonctions de l'acte d'imagination dans l'économie générale de la conscience (2). Après

${ }^{1}$ J.-P. Sartre, Lettres au Castor et à quelques autres, Paris, Gallimard, 1983, Tome 2, p. 115.

J.-P. Sartre, «Une idée fondamentale de la phénoménologie de Husserl: l'intentionnalité ", dans Id., La Transcendance de l'Ego et autres textes phénoménologiques, Paris, Vrin, 2003, p. 87-89. 
quoi, nous tenterons plus spécifiquement de préciser le rôle joué par l'acte d'imagination dans l'événement de la personnalisation de l'être-pour-soi (3). Enfin, nous prendrons appui sur ces analyses afin de commenter, dans notre conclusion, un point de l'histoire de la philosophie phénoménologique (4). Nos remarques conclusives viseront en effet à préciser, à la suite de Vincent de Coorebyter, "la manière dont Sartre comprend, exploite et dépasse la théorie husserlienne de l'image $»{ }^{1}$. De sorte que nous parcourrons, dans ce texte, les tensions reliant l'intentionnalité imaginative à l'ipséité de la conscience et, du point de vue de l'histoire de la philosophie, certaines des torsions que Sartre fit subir à la philosophie husserlienne de l'imagination.

\section{Pour une déduction des propriétés de l'intentionnalité imaginative}

« Le grand événement de la philosophie d'avant-guerre », affirme Sartre dans l'un de ses premiers portraits de Husserl, « est certainement la parution du premier tome de la Revue annuelle de philosophie et de recherches phénoménologiques qui contenait [...] Esquisse d'une phénoménologie pure et d'une philosophie phénoménologique ${ }^{2}$. Ce témoignage ne peut faire l'objet d'un doute. Les Ideen firent en effet date dans le paysage de la philosophie française. Cela étant, la compréhension sartrienne de l'œuvre de Husserl demeure problématique aux yeux de beaucoup. Du moins, comme le rappelle Vincent de Coorebyter, nombre de phénoménologues considèrent que Sartre a pris dans les Idées directrices «l'accessoire pour l'essentiel et l'essentiel pour l'accessoire $»^{3}$; c'est pourquoi son interprétation de ce texte peut être probablement reléguée au rang de ce que Paul Ricœur a appelé «l'histoire des hérésies husserliennes » ${ }^{4}$. Gérard Granel ne dit d'ailleurs pas autre chose lorsqu'il taxe de « bouffonnerie ${ }^{5}$ la filiation unissant les phénoménologies sartrienne et husserlienne.

Le commentaire de Jean Hyppolite discerne, quant à lui, un rapport de «subversion ${ }^{6}$ entre ces deux auteurs. Cette appréciation nous paraît particulièrement vraie concernant le concept d'intentionnalité. Comme le

\footnotetext{
${ }^{1}$ V. de Coorebyter, « De Husserl à Sartre. La structure intentionnelle de l'image dans L'Imagination et L'Imaginaire », Méthodos $\mathrm{n}^{\circ} 12$ (2012), p. 2.

2 J.-P. Sartre, L'Imagination, Paris, PUF, 2010, p. 139.

${ }^{3} \mathrm{~V}$. de Coorebyter, Sartre face à la phénoménologie, Bruxelles, Ousia, 2000, p. 34.

${ }^{4} \mathrm{P}$. Ricœur, « De la phénoménologie », dans Id., À l'école de la phénoménologie, Paris, Vrin, 1998, p. 156.

${ }^{5}$ G. Granel, Traditionis traditio, Paris, Gallimard, 1972, p. 20.

${ }^{6}$ J. Hyppolite, Figures de la pensée philosophique, Paris, PUF, 1972, p. 759.
} 
rappelle en effet Hyppolite, Sartre donne de cette notion « une interprétation qui lui est personnelle et qui déjà contient en germe les thèmes et le nœud même de sa propre philosophie ${ }^{1}$. Certes, «l'article sur l'intentionnalité » admet avec Husserl ce principe selon lequel «toute conscience est conscience de quelque chose $»^{2}$. Mais le texte indique également que ce « quelque chose » est extérieur à la conscience. Pour Sartre, toute conscience est, donc, consciente d'une extériorité qui n'est pas elle, consciente «d'autre chose que soi », révélation aussi de cette «chose» étrangère dont elle « souffre d'abord la qualité objective $»^{3}$. "La conscience ne précède jamais l'objet ${ }^{4}$, répètera-t-il encore dans L'Imaginaire. Dans ces conditions, l'intentionnalité désigne dès lors la relation directe et spontanée de la conscience à son dehors. Bien plus, la conscience n'étant que cette fuite vers une chose extérieure, il lui est impossible, en retour, de se replier sur ellemême dans le for d'une intériorité. Ainsi, pour «l'article sur l'intentionnalité », la conscience «n'a pas de dedans » ${ }^{5}$. Elle est donc un lieu vide, à l'inverse de la conception husserlienne de la vie mentale qui dépeint la conscience comme une jungle de vécus intentionnels ${ }^{6}$.

Sans pousser plus loin l'analyse de ce texte datant de $1934^{7}$, notons que celui-ci articule l'une à l'autre deux propositions philosophiques: la conscience est vide, d'une part, car, d'autre part, l'intentionnalité est une relation spontanée au monde. Ailleurs, nous avons fait l'hypothèse que ces deux thèses permirent à Sartre de construire la "preuve ontologique » de L'Étre et le Néant ${ }^{8}$. À présent, nous voudrions montrer que ces deux prémisses reconduisent également aux propriétés de l'acte d'imagination, décrites dans la première partie de L'Imaginaire. Nous souhaitons, en d'autres termes, faire l'hypothèse de lecture suivante: les propriétés de l'imagination procèdent, chez Sartre, d'une déduction des propositions philosophiques posées par «l'article sur l'intentionnalité ».

\footnotetext{
${ }^{1} I d$.

2 J.-P. Sartre, « L'intentionnalité », art. cit., p. 89.

${ }^{3}$ Ibid., pp. 88-89.

${ }_{5}^{4}$ J.-P. Sartre, L'Imaginaire, op. cit., p. 29.

${ }^{5}$ Ibid., p. 88.

${ }^{6} \mathrm{Cf}$. Le chapitre II de la cinquième des Recherches logiques.

${ }^{7}$ Pour une étude approfondie de ce texte, nous renvoyons aux travaux susmentionnés de J. Hyppolite et V. de Coorebyter.

${ }^{8}$ F. Recchia, «Émotion, ipséité, liberté : Réflexions à propos des fondements de la théorie sartrienne des émotions", Bulletin d'analyse phénoménologique XII 5 (2016), pp. 3-8.
} 
Dans L'Imaginaire, la «spontanéité» ${ }^{1}$ est épinglée comme l'une des principales caractéristiques de l'acte d'imagination. Or, on l'aura compris, cette propriété dévoile l'existence d'une analogie entre, d'une part, l'intentionnalité en général et, d'autre part, la spécification de celle-ci en une intentionnalité imaginative. Plus largement, cette analogie révèle qu'un progrès significatif est réalisé par l'œuvre sartrienne, entre 1934 et 1940. L'Imaginaire, selon nous, particularise, autant qu'il précise, la deuxième des deux thèses de «l'article sur l'intentionnalité ». Le texte de 1940 apporte en effet quelques indications supplémentaires concernant la spontanéité qui caractérise en propre l'acte d'imagination. Il s'agit d'une "spontanéité qui produit et conserve l'objet en image $»^{2}$. D'où il suit que, d'un texte à l'autre, Sartre infléchit durablement sa théorie de l'intentionnalité en direction d'une analyse de la production-conservation d'objets imaginaires.

La deuxième des propriétés de l'acte d'imagination concerne l'image, qui est le produit de l'intention imaginative. Sur ce point, L'Imagination a noté de bonne heure que la théorie de l'intentionnalité était "appelée à renouveler la notion d'image $»^{3}$. C'est que, l'intention imaginative n'étant qu'un rapport au monde, il s'ensuit que son produit, l'image, " ne saurait [...] désigner que le rapport de la conscience à l'objet $\gg{ }^{4}$. De sorte que l'image n'est pas un portrait mental de l'objet, rangé quelque part dans la conscience à côté d'autres "petits simulacres ${ }^{5}$ de la réalité mondaine. Sartre soutient qu'elle est au contraire « une certaine façon qu'a l'objet d'apparaître à la conscience, ou, si l'on préfère, une certaine façon qu'a la conscience de se donner un objet $»^{6}$. Or, selon nous, cette définition de l'image constitue, là encore, un approfondissement de «l'article sur l'intentionnalité ». Car, en précisant une nouvelle fois la seconde thèse de ce texte, l'œuvre sartrienne peut soutenir que l'intentionnalité imaginative est une relation au monde qui permet la transformation de ses objets en image.

Plus bas, nous tenterons de comprendre la manière dont procède cette transformation. Pour l'heure, considérons cette nouvelle caractéristique de l'acte d'imagination: l'image, corrélat d'une intention imaginative, "pose son objet comme un néant ${ }^{7}$. En effet, alors que la perception livre son objet

\footnotetext{
${ }^{1}$ J.-P. Sartre, L'Imaginaire, op. cit., p. 35.

${ }^{2}$ Idem. Nous soulignons.

${ }^{3}$ J.-P. Sartre, L'Imagination, op. cit., p. 144.

${ }^{4}$ J.-P. Sartre, L'Imaginaire, op. cit., p. 21.

${ }^{5}$ Ibid., p. 17.

${ }^{6}$ Ibid., p. 21.

${ }^{7}$ Ibid., p. 33.
} 
par des myriades d'Abschattungen, "l'objet de l'image n'est jamais rien de plus que la conscience qu'on en a $»^{1}$. Créés sous l'effort d'une intention, les objets imaginaires «n'existent pour autant qu'on les pense $»^{2}$. Contemporains de la conscience que nous en prenons, dit encore Sartre, ils sont marqués d'une "pauvreté essentielle » ${ }^{3}$. Ici, L'Imaginaire rejoint peut-être les analyses de Descartes qui, dans la sixième des Méditations métaphysiques, remarque qu'imaginer un chiliogone suppose « une particulière contention d'esprit $\gg{ }^{4}$. De sorte qu'il est impossible de porter concomitamment son attention sur les milles côtés de ce polygone. De son côté, Sartre réitère ce constat en indiquant que l'acte d'imagination peut tout au plus permettre de discerner « deux ou trois rapports $»^{5}$ dans l'objet qui apparait en image. Ainsi, chiliogone ou pas, l'objet-image se donne comme un néant d'être, tant est ténue l'expérience que nous en faisons par le biais de l'intentionnalité imaginative.

Dans l'introduction de L'Imagination, Sartre voyait même dans cette légèreté la caractéristique définissant la forme d'existence particulière de l'image. On peut en effet reconnaître celle-ci au fait qu'elle «ne s'impose pas comme une limite ${ }^{6}$ à la conscience, au contraire des choses perçues qui « échappent à la domination de la conscience » ${ }^{7}$ en raison de leur présence et de leur inertie. Plus profondément encore, dans L'Imaginaire, cette vacuité intrinsèque de l'objet en image renvoie à un type particulier d'attitude phénoménologique : celui de la «quasi-observation ${ }^{8}$, soit la posture de notre conscience face aux images et dont Sartre remarque qu'elle forme la quatrième et dernière propriété de l'acte d'imagination. Selon lui, l'intention imaginative produit un objet que nous pouvons observer à loisir. Mais cette observation n'apprend rien sur l'objet, ou alors vraiment très peu de choses, parce qu'elle permet de discerner peu de rapports entre les différentes facettes de l'objet figuré en image. En ce sens, les descriptions de L'Imaginaire s'inscrivent peut-être encore une fois dans la continuité des analyses cartésiennes montrant que l'imagination « ne sert en aucune façon à

\footnotetext{
${ }^{1}$ Ibid., p. 27.

${ }^{2}$ Ibid., p. 26.

${ }^{3}$ Idem L'Imagination.

${ }^{5}$ J.-P. Sartre, L'Imaginaire, op. cit., p. 28.

${ }^{6}$ J.-P. Sartre, L'Imagination, op. cit., p. 2.

${ }^{7}$ Idem

${ }^{8}$ J.-P. Sartre, L'Imaginaire, op. cit., p. 28.
}

${ }^{4}$ R. Descartes, Méditations métaphysiques, Paris, GF Flammarion, 2011, AT 57, p.

177. Sartre récupère d'ailleurs ce philosophème cartésien dans l'introduction de 
découvrir les propriétés qui font la différence du chiliogone d'avec les autres polygones $»^{1}$. Ainsi, tout ce que peut l'intentionnalité imaginative, c'est rendre présent un objet sur le mode du presque vécu. Elle le présente d'ailleurs très curieusement «du dehors et du dedans à la fois ${ }^{2}$, remarque Sartre. L'objet est observé du dehors, car l'acte d'imagination s'enracine dans un rapport au monde. Mais l'objet corrélatif de cet acte, l'image qui émerge à partir du monde, n'est, selon Sartre, pas autre chose qu'une conscience imageante qui produit cet objet. Si bien que la conscience examine un objet du monde au travers l'image qu'elle s'en fait. De là toute l'ambigüité des vécus que nous éprouvons au travers de l'imagination : ils nous mettent en face d'un objet, tandis qu'ils nous font également prendre place en lui.

L'image pose son objet comme un néant, et, de ce fait, l'acte d'imagination entraine l'attitude phénoménologique sui generis de la quasiobservation - telles sont les caractéristiques de l'intentionnalité imaginative que, dans L'Imaginaire, Sartre découvre à partir de la réflexion. Or, conformément à notre hypothèse, il nous semble que ces propriétés peuvent être également comprises comme des conséquences découlant de la vacuité de la conscience, soit la thèse défendue par «l'article sur l'intentionnalité ». Force est en effet de constater que la conscience imageante est évidée à l'instar de la conscience " claire comme un grand vent $)^{3}$ décrite par l'article de 1934. Une fois encore, il existe un rapport d'analogie entre la vacuité de la conscience, en général, et celle qui anime, en particulier, la conscience imageante. De cette manière, les images posent leurs objets intentionnels sur le mode d'un néant d'être, c'est-à-dire d'une absence, qui rappelle in fine la vacuité originaire de la conscience. En retour, la conscience imageante peut même apparaître, dans l'œuvre sartrienne, comme la modalité paradigmatique de la conscience intentionnelle, dans la mesure où elle en illustre à la perfection le caractère évidé. Plus largement encore, quoique de manière anticipée et quelque peu abstraite, la dernière ligne de l'article de 1934 semble développer l'idée d'une posture phénoménologique quasi-observationnelle. "Ce n'est pas dans je ne sais quelle retraite que nous nous découvrirons », affirme en effet le texte de 1934, "c'est sur la route, dans la ville au milieu de la foule, chose parmi les choses, homme parmi les hommes $»^{4}$. Au-delà de l'affirmation d'une transcendance radicale de la

\footnotetext{
${ }^{1}$ R. Descartes, Méditations métaphysiques, op. cit., AT 57, p. 177.

2 J.-P. Sartre, L'Imaginaire, op. cit., p. 29.

${ }^{3}$ J.-P. Sartre, « L'intentionnalité », art. cit., p. 88.

${ }^{4}$ Ibid., p. 89.
} 
conscience, ce passage indique aussi, d'après nous, que la conscience se découvre au milieu du monde en tant qu'elle est un rapport à ce monde même. En ce sens, elle se tient face à son objet parce qu'elle s'y rapporte spontanément; mais c'est d'abord en vivant au travers de lui qu'elle peut découvrir qu'elle s'y rapporte. Il faut, en d'autres termes, que la conscience soit à l'intérieur de son objet pour constater son extériorité dans un examen réflexif. Elle est, pour le dire encore autrement, présente au monde du dehors et du dedans à la fois. C'est pourquoi nous pouvons faire l'hypothèse que la conscience se tient toujours déjà dans un rapport de quasi-observation avec le monde. Quant à l'acte d'imagination, il reproduit cette attitude phénoménologique à l'égard d'une variété particulière de produits de la conscience : ses images.

En résumé, les descriptions de la première partie de L'Imaginaire indiquent que l'acte d'imagination peut être caractérisé comme suit : il s'agit d'une négation spontanée qui pose en image un objet pauvre de contenu et qui, en outre, s'y rapporte sur le mode d'une quasi-observation. Pour notre part, nous avons tenté de montrer comment chacune de ces propriétés prolongeait les prémisses philosophiques posées par «l'article sur l'intentionnalité ». C'est pourquoi nous pensons que le rapport entre ces deux textes est celui d'une déduction. Mais le concept de déduction auquel nous nous référerons n'est pas celui de la logique formelle. En effet, nous n'affirmons pas que les analyses de L'Imaginaire sont des conclusions déjà enfermées dans le texte de 1934 et qui préservent sa vérité. Nous lisons plutôt les deux textes à l'aune du principe kantien de la déduction, à savoir une «preuve [...] qui doit faire paraître le droit ou la légitimité» ${ }^{1}$ des concepts. Tout ce que nos remarques visaient ainsi à souligner, c'est que la description sartrienne de l'acte d'imagination est légitime, non en raison d'une méthode réflexive peu précise et qui débouche sur une conception usurpée de la conscience imageante, mais bien parce qu'elle s'inscrit dans le prolongement et sur le fond d'une théorisation originale de l'intentionnalité.

\section{De l'irréalité à la liberté : les fonctions de l'intentionnalité imaginative}

Intéressons-nous maintenant aux fonctions spécifiques assurées par l'acte d'imagination dans l'économie générale de la conscience. Plus précisément,

${ }^{1}$ I. Kant, Critique de la raison pure, trad. fr. A. J.-L. Delamarre et F. Marty, Paris, Gallimard, 1980, Analytique transcendantale, § 13, A 84, p. 148. Nous soulignons. 
examinons tout d'abord en quoi consiste le travail par lequel l'intentionnalité imaginative produit un objet en image. Rappelons à cette fin que, pour Sartre, « la conscience d'image est une forme synthétique qui apparait comme un certain moment d'une synthèse temporelle et s'organise avec d'autres formes de conscience [...] pour former une unité $»^{1}$. L'imagination, en d'autres termes, ne peut être isolée d'un flux de conscience, où son action consiste à synthétiser un nouveau vécu sur la base d'Erlebnisse plus anciennes. "Produire en moi la conscience imageante de Pierre», affirme en ce sens L'Imaginaire, "c'est faire une synthèse intentionnelle qui ramasse en elle une foule de moments passés $»^{2}$. Dès lors, le problème essentiel auquel nous devons nous attacher est celui de la description des moments composant cette liaison synthétique.

Pour Sartre, la synthèse intentionnelle produisant des images consiste à réaliser un savoir dans une matière intuitive ${ }^{3}$. Autrement dit, l'acte d'imagination met en relation des données perceptives et intellectuelles. Par un jeu de rétention, il se saisit d'abord de la matière intuitive d'une perception. Mais, simultanément, il applique sur ces données intuitives une couche de concepts. Ce qui a une incidence sur l'objet intentionnel présenté à la conscience au travers de cette matière intuitive. Car, pénétrant l'intuition de concepts, l'acte d'imagination élève l'objet présenté par cette diversité «à l'état de matière représentative ${ }^{4}$ autonome. D'où il suit que l'imaginaire reconstitue, d'un côté, un nouvel objet sur la base d'un objet perçu ; tandis que, d'un autre côté, cet objet imaginaire joue le rôle d'un représentant de l'objet perçu. Bien plus, l'œuvre sartrienne articule deux conséquences à cette description de la synthèse de l'imagination. D'une part, en réactivant le vocabulaire de la phénoménologie husserlienne, Sartre soutient que l'objet imaginaire est un «analogon $»^{5}$ de l'objet perçu, dans la mesure où l'image entretient une certaine ressemblance avec la chose qu'elle représente. D'autre part, cette description de l'imagination ressurgit dans une thèse qui concerne la pensée conceptuelle. S'adossant à sa description de l'image comme mixte de concepts et d'intuitions, L'Imaginaire soutient en effet que la synthèse intentionnelle imaginaire rend possible "une incarnation de la pensée ${ }^{6}$

\footnotetext{
${ }^{1}$ J.-P. Sartre, L'Imaginaire, op. cit., p. 37.

${ }^{2}$ Ibid., p. 34.

${ }^{3}$ Ibid., p. 59.

${ }^{4}$ Ibid., p. 58.

${ }^{5}$ Ibid., p. 51.

${ }^{6}$ Ibid., p. 216.
} 
dans une matière intuitive. Car la pensée prend, pour ainsi dire, corps dans la matière intuitive composant l'image.

Toutefois, on l'aura compris, une ambigüité traverse cette description de la liaison imaginaire. Elle concerne la manière dont l'imagination appréhende les données intuitives de la conscience. Celles-ci sont, d'un côté, réactivées dans une nouvelle organisation qui est l'image. Mais, d'un autre côté, elles subissent une altération dans la mesure où l'imagination mélange des contenus perceptifs et conceptuels, créant ainsi des images allégées en intuition puisque celles-ci «ne peuvent se constituer qu'au prix de l'anéantissement des consciences perceptives ${ }^{1}$. Cela étant, la phénoménologie sartrienne de l'image ne voit pas dans cette ambigüité descriptive un problème. Elle y décèle plutôt la fonction principale de l'acte d'imagination : celui-ci assure, en effet, une fonction d'irréalisation des données perceptives à partir de pensées conceptuelles. Quant à ces pensées qui s'incarnent dans la matière de l'image, elles permettent, en contrepartie, que la conscience croie à ses images et puisse, par la même, mener une vie imaginaire.

Loin de représenter un événement fugace ou nocturne de l'existence, pour Sartre, cette vie en imagination occupe une place considérable dans le champ de la conscience. Elle peut même supplanter la vie réelle. Du moins, cette dernière idée traverse L'Idiot de la famille. L'analyse progressiverégressive de Flaubert dévoile en effet que la personnalisation de l'auteur de Madame Bovary dépend très étroitement de la capacité de ce dernier à écouler, à l'aide notamment de la littérature, l'espace du réel dans un univers d'images ${ }^{2}$. De même, le troisième des Carnets de la drôle de guerre souligne la contribution apportée par l'imaginaire dans la mise en place de projets au sein d'une situation marquée par un conflit mondial où toutes perspectives semblent anéanties. Pour vivre et être dans une guerre, il faut que la conscience puisse s'évader de cette situation conflictuelle. Il faut qu'elle puisse se plonger, dit Sartre, dans un état de «confiance magique ${ }^{3}$ où les batailles apparaissent comme un jeu.

Or, nous voudrions suggérer que, par-delà cette fonction d'irréalisation de l'imagination qui apparaît fort documentée dans l'œuvre sartrienne, l'acte d'imagination assure également une seconde fonction qui est plus discrètement thématisée par Sartre. Bien qu'il ne l'affirme jamais explicitement dans L'Imaginaire, tout s'y passe comme si l'imagination jouait dans l'économie

\footnotetext{
${ }^{1}$ Ibid., p. 234.

${ }^{2}$ Cf. J.-P. Sartre, L'Idiot de la famille, Paris, Gallimard, 1988, p. 653-665.

${ }^{3}$ J.-P. Sartre, Carnets de la drôle de guerre, dans Id., Les Mots et autres écrits autobiographiques, Paris, Gallimard, 2010, p. 355.
} 
globale de la conscience le rôle d'un instrument de persuasion qui motive le sujet à agir au sein de sa situation. Roland Breeur a fort justement épinglé ce rôle fonctionnel de l'imagination. Il a en effet remarqué que celle-ci contracte

une sorte de pacte quasi magique avec les choses, une parenté : celles-ci prennent peu à peu mes traits, moi-même devenant peu à peu ce creux où se concentre une conscience qui s'échappe vers cette épaisseur qu'est le monde ${ }^{1}$.

Dans L'Imaginaire, nous l'avons dit, l'imagination anéantit le monde qu'elle reconstruit dans une image. Laquelle est, pour prolonger l'analyse de Roland Breeur, taillée à la mesure des désirs du sujet. La conscience peut, dès lors, y reconnaître des présages qui forment pour elle autant d'invitations à s'échapper vers le monde au travers d'actions. Par voie de conséquence, en nous appuyant sur la relecture breeurienne de L'Imaginaire, nous voudrions souligner que la fonction d'irréalisation de l'imagination est doublée d'une fonction de persuasion. Car l'intentionnalité imaginative convainc pour ainsi dire la subjectivité sartrienne de la nécessité de son action. Cela, notamment, en établissant dans la situation un «couloir» d'actions possibles qui est mis en place, non pas de manière concrète à partir d'un dispositif d'ustensiles balisant la voie d'une action à venir, mais bien seulement au moyen d'une représentation de la situation, par le biais d'une image de la réalité, qui présente un portrait du monde-de-la-vie facilitant la mise en route d'un projet pratique.

Nous comprenons par là-même que l'imagination assure chez Sartre, un levier pour l'action. Or, dans le contexte d'une œuvre où la liberté est posée comme la «condition indispensable et fondamentale de toute action $»^{2}$, l'intentionnalité imaginative enveloppe donc quelque chose de l'ordre d'une fonction de libération. En ce sens, la conclusion de L'Imaginaire démêle l'écheveau de relations unissant la liberté du sujet à son imagination. Sartre y cherche en effet à identifier les conditions de possibilité d'une conscience imageante, lorsqu'il demande ce «que doit être la conscience en image s'il est vrai qu'une constitution d'image doit toujours être possible $»^{3}$. Plus précisément, l'enjeu impliqué par une telle interrogation consiste à déterminer si l'imagination est « une spécification contingente et métaphysique de l'essence "conscience" » ou bien, au

\footnotetext{
${ }^{1}$ R. Breeur, Autour de Sartre. La conscience mise à nu, Grenoble, Millon, 2005, p. 146.

${ }^{2}$ J.-P. Sartre, L'Être et le Néant, op. cit., p. 480.

${ }^{3}$ J.-P. Sartre, L'Imaginaire, op. cit., p. 343.
} 
contraire, si elle doit être décrite « comme une structure constitutive de cette essence ${ }^{1}$. À l'issue de cette discussion, Sartre dégage une condition essentielle de l'acte d'imaginer: "Pour qu'une conscience puisse imager», dit-il, « il faut qu'elle ait la possibilité de poser une thèse d'irréalité $»^{2}$. Ce qui revient à constater que l'imagination, en sa structure la plus intime, est une négation. Mieux encore, celle-ci enveloppe à la fois un rapport à la réalité et une néantisation de celle-ci. Cela tant et si bien que L'Imaginaire conclut en indiquant que ce mouvement de « constitution et néantisation du monde $»^{3}$, ce mouvement qui caractérise spécifiquement le déroulement d'une intentionnalité imaginative, n'est pas simplement une modalité particulière de conscience, mais bien, au contraire, «la conscience toute entière en tant qu'elle réalise sa liberté ${ }^{4}$ en situation. C'est en effet

l'apparition de l'imaginaire devant la conscience qui permet de saisir la néantisation du monde comme sa condition essentielle et comme sa structure première. S'il était possible de concevoir un instant une conscience qui n'imaginerait pas, il faudrait la concevoir comme totalement engluée dans l'existant et sans possibilité de saisir autre chose que de l'existant. Mais précisément c'est ce qui n'est pas ni ne saurait être : tout existant dès qu'il est posé, est dépassé par là-même. Mais encore faut-il qu'il soit dépassé vers quelque chose. L'imaginaire est en chaque cas le "quelque chose » concret vers quoi l'existant est dépassé ${ }^{5}$.

Ainsi l'imagination n'est donc pas une spécification contingente de la conscience. Elle en est au contraire le cas paradigmatique, car cet acte intentionnel met en exergue la structure profonde de la conscience : celle d'une négation qui est l'envers de la liberté du sujet. En ce sens, comme nous l'affirmions plus haut, la phénoménologie de l'image sartrienne débouche sur une philosophie consistante de la liberté. Celle-ci porte, de manière plus précise, sur le concours apporté par l'imagination durant les processus de libération du sujet. L'Être et le Néant développe en effet cette idée admirable, selon nous - que la libération d'une situation ne devient possible qu'une fois seulement imaginée la perspective de son dépassement. Voyons, par exemple, le cas de l'ouvrier de 1830 qui est

\footnotetext{
${ }^{1}$ Ibid., p. 344.

${ }^{2}$ Ibid., p. 351.

${ }^{3}$ Ibid., p. 357.

${ }^{4}$ Ibid., p. 358.

${ }^{5}$ Ibid., p. 359.
} 
plongé dans la situation historique, il lui arrive de ne même pas concevoir les défauts et les manques d'une organisation politique [...] parce qu'il la saisit dans sa plénitude d'être et qu'il ne peut même imager qu'il puisse en être autrement. [...] L'ouvrier de 1830 [...] ne se représente pas ses souffrances comme intolérables, il s'en accommode, non par résignation, mais parce qu'il manque de la culture et de la réflexion nécessaires pour lui faire concevoir un état social où ces souffrances n'existeraient pas. Aussi, n'agit-il pas. [...] Mais tout au contraire, c'est lorsqu'il aura fait le projet de [...] changer [sa situation] qu'elle lui paraitra intolérable ${ }^{1}$.

La liberté de l'ouvrier ne devient donc effective qu'au travers d'une action menée en situation; mais cette action se produit si une intention imaginative a, par avance, constitué la finalité de l'agir. De sorte que l'une des fonctions de l'acte d'imagination, peut-être même sa fonction principale, et dont Sartre n'a pas jugé bon de réaliser une étude systématique séparée, consiste à garantir la libération du sujet. Ce qui élève au nombre de trois les fonctions de l'intentionnalité imaginative. Celle-ci assure, en résumé, une irréalisation entrainant l'anéantissement d'une matière intuitive, mais aussi la possibilité d'une vie imaginaire. Ce à quoi s'ajoute une fonction de persuasion, puisque l'imagination produit un analogon de la situation qui conforte la conscience dans sa résolution d'agir. Enfin, nous venons de lire comment l'œuvre sartrienne assigne une fonction de libération à la conscience imageante. Et nous voudrions à présent prolonger les analyses de Sartre, en mettant en évidence le rôle que jouent ces fonctions au cours du processus de la personnalisation de la conscience.

\section{Imagination et singularité}

Dans le champ des études sartriennes, de récents travaux ont mis en évidence que la philosophie sartrienne est traversée par le problème de la personnalisation. Dépassant le cadre fixé par la conclusion de La Transcendance de l'Ego qui définit la conscience comme un champ transcendantal impersonnel $^{2}$, Jean-Marc Mouillie et Hadi Rizk ${ }^{3}$ ont notamment montré que $L$ 'Etre et

${ }^{1}$ J.-P. Sartre, L'Être et le Néant, op. cit., p. 479.

2 J.-P. Sartre, La Transcendance de l'Ego, Paris, Vrin, 2012, p. 79.

${ }^{3}$ J.-M. Mouillie, Sartre : Conscience, Ego et Psyché, PUF, 2000 ; H. Rizk, «Être et faire, la liberté comme principe d'individuation », dans J.-M. Mouillie et J.-P. Narboux (dir.), Sartre. L'Être et le néant. Nouvelles lectures, Paris, Les Belles Lettres, 2015, p. 193-212. 
le Néant prolonge la critique sartrienne de la catégorie de la personnalité dans une théorie de l'ipséité de la conscience. En ce sens également, Vincent de Coorebyter et Juliette Simont ${ }^{1}$ ont identifié le «recueil de Corbin» comme la pièce philosophique qui a permis à l'œuvre sartrienne de passer de la critique d'une catégorie conceptuelle à l'élaboration de sa propre théorie de la personne. Sartre s'adosse en effet à Heidegger et son analyse de la Selbstheit du Dasein ${ }^{2}$ pour élaborer, dans L'Etre et le Néant, une théorie du « circuit de l'ipséité » ${ }^{3}$ de la conscience.

Dans l'ontologie phénoménologique de 1943, cette théorie de l'ipséité trouve son ancrage initial dans l'interaction entre l'être-pour-soi et l'être-ensoi. L'être-pour-soi, c'est-à-dire «l'être du sujet » ou plus simplement "la conscience », rappelons-le, se rapporte intentionnellement à l'en-soi qui désigne la région d'être occupée par le monde. Mais le pour-soi est pourvu de ce que Sartre appelle un "néant interne ${ }^{4}$. Il s'agit d'une insuffisance d'être en raison de laquelle la conscience se rapporte au monde sur le mode d'une négation. La conscience se rapporte ainsi à l'état de choses de la situation; elle le nie et elle le dépasse pour mettre en place un nouvel état de choses possible. Mais cette nouvelle possibilité, nous l'avons appris du cas de l'ouvrier de 1830, est une production imaginaire de la conscience. De sorte que la conscience vise, en définitive, ses propres objets intentionnels. Pour mieux dire, puisque la phénoménologie sartrienne soutient la thèse d'une identité entre la conscience et ses contenus intentionnels, ce à quoi la conscience se rapporte lorsqu'elle vise ce contenu possible, c'est une version possible d'elle-même. Or, "ce rapport du pour-soi avec le possible qu'il est», soutient Sartre dans L'Etre et le Néant, il faut l'appeler «circuit de l'ipséité 》".

Il convient en effet de parler d'un « circuit », dans la mesure où la trajectoire globale de l'intentionnalité de la conscience prend la forme d'un parcours entre elle-même hic et nunc et elle-même comme possible, tel que l'un et l'autre points de cette trajectoire sont séparés par le monde, qui jouxte la conscience présente et dont le dépassement par une négation intentionnelle conduit à la conscience possible. De manière plus rigoureuse, nous pouvons

\footnotetext{
${ }^{1}$ V. de Coorebyter, Sartre face à la phénoménologie, op. cit., p. 570 ; J.-P. Sartre, Carnets de la drôle de guerre, op. cit., p. 1394 (note de J. Simont).

${ }^{2}$ M. Heidegger, Ce qui fait l'être-essentiel d'un fondement ou « raison », trad. fr. H. Corbin, dans Id., Questions I \& II, Paris, Gallimard, 1968, p. 23-84.

${ }^{3}$ J.-P. Sartre, L'Être et le Néant, op. cit., pp. 139-141.

${ }^{4}$ Ibid., p. 56 et 114.

${ }^{5}$ Ibid., p. 139.
} 
décomposer ce circuit en trois pièces reliées entre elles par l'arc intentionnel de la conscience : la " facticité ${ }^{1}$ de l'être-pour-soi, désignant pour Sartre l'ensemble des déterminations contingentes qu'est de facto le sujet; le possible projeté, vers lequel converge la conscience; et le monde, qui est «l'obstacle nécessaire $»^{2}$ traversé pour atteindre ce possible. Ensemble, ces trois éléments forment un circuit de «l'ipséité ». Car cette boucle intentionnelle entraine un rapport de la conscience à une image possible d'elle-même, qui est d'après Sartre le soi de la conscience ${ }^{3}$. De sorte que, en résumé, l'intentionnalité de la conscience implique, dès $L$ 'Etre et le Néant, un rapport à soi, que Sartre appelle "circuit de l'ipséité " et sur la base duquel il développe une théorie de la personnalisation de la conscience à partir de son intentionnalité, mais aussi une méthodologie permettant d'analyser cette personnalisation : la psychanalyse existentielle.

Bien plus, cette théorie de l'ipséité enveloppe un thème de réflexion qui s'étend dans l'œuvre sartrienne jusqu'à L'Idiot de la famille : celui de la singularité du sujet définie comme un équilibre mouvant entre la contingence qu'on est, le monde qu'on vit, et le possible qu'on vise. Le circuit de l'ipséité est en effet une structure instable, dans la mesure où il peut être déséquilibré par les termes qu'il relie. Cela est particulièrement vrai concernant la facticité et le monde. Respectivement point de départ et milieu conducteur du circuit de l'ipséité, l'un et l'autre y créent des points de tension; car ils opposent à la conscience des résistances empêchant celle-ci de performer le projet intentionnel qui entraine sa personnalisation. Cela étant dit, il nous semble que, dans l'œuvre sartrienne, l'imagination intervient précisément pour amincir ces résistances. Plus précisément, c'est la production d'objets imaginaires qui permet une telle neutralisation.

Pour illustrer cette idée, reconsidérons le cas de Flaubert. Dès «les premières lettres de sa Correspondance, nous apprenons qu'il veut écrire $»^{4}$. Cela étant, des difficultés ont compliqué ce projet dès le départ. "Longtemps Gustave n'a pu saisir les liaisons élémentaires qui font de deux lettres une syllabe, de plusieurs syllabes un mot. [...] Donc, Gustave était en retard sur toute la ligne. [...] Et le doute commença à naitre : Gustave n'est-il pas un idiot? $»^{5}$. Sur le plan phénoménologique, ce qui pose problème à la conscience flaubertienne, c'est une hypersensibilité perceptive à la phoné-

\footnotetext{
${ }^{1}$ Ibid., p. 119.

${ }^{2}$ Ibid., p. 141.

${ }^{3}$ Ibid., p. 113.

${ }_{5}^{4}$ J.-P. Sartre, L'Idiot de la famille, op. cit., p. 659.

${ }^{5}$ Ibid., p. 14.
} 
tique du langage. Pour elle, tout se passe comme si «le langage n'était [...] que des bruits qui parlent $»^{1}$. Comprenez: pour Flaubert qui fait le projet d'écrire, tous les mots sont difficiles. Mais c'est sans compter sur son imagination. « $[\grave{\mathrm{A}}]$ treize ans, Gustave, lui tout seul, compose un journal littéraire $»^{2}$. C'est que, dès la puberté, «Flaubert associe Vie et Destin, Souffrance et Châtiment, Fausse Mort et Survie $»^{3}$. En bref, son imagination complique les signifiants du langage en symboles. La difficulté des mots est alors dépassée dans un objet irréel: un système symbolique permettant à Flaubert d'anéantir la matière phonétique du langage, mais aussi, et surtout, de mettre en place le projet d'écrire qui va entrainer sa personnalisation. En effet, dès sa petite adolescence, Flaubert « se projette dans ses nouvelles et s'y fait un Autre, sans bien comprendre son entreprise $»^{4}$. Ainsi se trouve déjà amorcée la trajectoire qui transformera l'idiot de la famille en génie de la littérature.

Dans ces conditions, il apparaît que, dans le cas de Flaubert, mais aussi, plus largement, dans l'œuvre sartrienne, l'imagination joue le rôle d'un stabilisateur de l'ipséité du pour-soi, dans la mesure où cette variété d'intentionnalité intervient comme une réponse adaptée aux contraintes opposées par la situation et la facticité. Mais le rôle de l'intentionnalité imaginative ne se limite pas seulement à la maintenance de ce circuit. Au contraire, comme nous venons encore de le voir avec L'Idiot de la famille, la conscience imageante participe pleinement à la constitution de la personnalisation du sujet. Le circuit de l'ipséité définit en effet le rapport du poursoi à ce possible qu'il est. Mais L'Être et le Néant, contre Leibniz, n'accorde pas au possible une réalité absolue ${ }^{5}$. Pour Sartre, un possible ne devient par conséquent possible qu'à la condition d'être posé par une conscience au travers d'une négation de l'état de choses situationnel. Or, dès la conclusion de L'Imaginaire, l'intentionnalité imaginative est présentée comme l'acte au fondement de cet anéantissement de la situation. Elle est même décrite par Sartre comme

un acte magique. C'est une incantation destinée à faire apparaître l'objet auquel on pense, la chose qu'on désire, de façon qu'on puisse en prendre

\footnotetext{
${ }^{1}$ Ibid., p. 22.

${ }^{2}$ Ibid., p. 325.

${ }^{3}$ Ibid., p. 328.

${ }^{4}$ Ibid., p. 329.

${ }^{5}$ J.-P. Sartre, L'Être et le Néant, op. cit., p. 133.
} 
possession. Il y a, dans cet acte, toujours quelque chose d'impérieux et d'enfantin, un refus de tenir compte de la distance, des difficultés ${ }^{1}$.

C'est dire, en somme, que l'imagination est une variété d'intentionnalité dont le rôle consiste à faire apparaître, dans la matière même de la situation, le possible appréhendé par le pour-soi dans son projet. De sorte que l'intentionnalité imaginative joue un rôle essentiel dans la personnalisation de la conscience, en ce sens qu'elle en assure la création à partir d'une situation, mais aussi le maintient contre une série de résistances. À la vérité, ce qui est finalement en jeu pour l'ouvrier qui imagine de nouvelles perspectives en 1830 , c'est donc l'expression de sa liberté au travers d'une lutte contre les déterminants socio-historiques de sa situation, autant que la consolidation de sa personne par ces mêmes déterminations qu'il vient éclairer à la lumière de son imagination.

\section{Conclusion : reprise et dépassement de l'analyse husserlienne de l'image}

La dette de Sartre à l'égard de Husserl est immense, et elle apparait très visiblement dans L'Imaginaire. Le projet sartrien d'une phénoménologie de l'image s'inscrit, en effet, dans le prolongement de la voie d'analyse ouverte par les appendices des paragraphes onze et vingt de la cinquième des Recherches logiques ${ }^{2}$. Husserl y démontre, contre Twardowski, que l'image comporte une structure intentionnelle ; de sorte qu'une analyse de l'imagination entraine, en contrepartie, " une explication du fonctionnement de l'intentionnalité, compte tenu du fait même que l'image désigne une certaine modalité intentionnelle $»^{3}$. Et, pour Vincent de Coorebyter, c'est à partir du paragraphe cinquante-trois des Idées directrices que Sartre a récupéré définitivement cette thèse selon laquelle l'image possède une structure intentionnelle ${ }^{4}$. Quoi qu'il en soit, pour l'auteur de L'Imaginaire, autant que pour celui des Ideen I, l'image n'est pas une chose occupant la conscience, ni une copie pure et simple de la perception. Il s'agit au contraire d'un acte qui restitue aux objets imaginaires leur caractère transcendant ${ }^{5}$. C'est pourquoi

${ }^{1}$ J.-P. Sartre, L'Imaginaire, op. cit., p. 239.

${ }^{2}$ Cf. E. Husserl, Recherches logiques, op. cit., Tome II/2, Cinquième recherche, pp. 281-231.

3 A. Hervy, "Perception et imagination: La problématique des actes mixtes », Bulletin d'analyse phénoménologique IX 1 (2013), p. 3.

${ }^{4} C f$. V. de Coorebyter, « De Husserl à Sartre », art. cit., p. 2.

${ }^{5}$ J.-P. Sartre, L'Imagination, op. cit., p. 147. 
aussi, de Husserl à Sartre, la phénoménologie de l'imagination coïncide avec une critique de la psychologie qui profite du concept d'image pour développer une conception immanentiste de la conscience. Enfin, d'un point de vue plus technique, dès L'Imaginaire et jusque dans L'Idiot de la famille, l'œuvre sartrienne récupère, dans la boîte à outils husserlienne, cette idée que l'image est un analogon par le biais duquel la conscience vise un objet.

Cela étant, dès L'Imagination, Sartre identifie deux lacunes dans l'analyse husserlienne de l'image ${ }^{1}$. D'une part, il y manque une description définissant les propriétés qui caractérisent en propre l'acte d'imagination, par rapport à d'autres variétés d'acte intentionnel. D'autre part, le statut de la matière de l'image mentale n'y est pas clair. En conséquence, comme le souligne Vincent de Coorebyter, l'on s'attendait à ce que L'Imaginaire se présente dans une " parfaite continuité ${ }^{2}{ }^{2}$ avec L'Imagination et apporte une solution à ces deux problèmes en donnant un commentaire pointu de l'analyse husserlienne de l'image. Or, c'est justement à ce niveau que se complique le rapport entre les œuvres husserlienne et sartrienne. L'Imaginaire " ne cite Husserl qu'à sept reprises en tout et pour tout ${ }^{3}$. L'analyse sartrienne de l'image prend au contraire ses appuis initiaux sur la philosophie cartésienne, et, sur le fond, si l'on en croit notre hypothèse, sur sa propre théorie de l'intentionnalité. L'Imaginaire développe ainsi une phénoménologie de l'image, " concurrente, voir adverse » ${ }^{4}$ à celle de Husserl, où Sartre ne cherche pas véritablement de solution aux problèmes qui traversent l'analyse husserlienne. Le rendez-vous est donc manqué entre les deux auteurs. Ce qui trahit que le texte de 1940 ne se limite pas à une reprise de la philosophie husserlienne. Il s'agit au contraire pour Sartre d'en opérer le renouvellement. Dans ses Carnets de la drôle de guerre, ce dernier ne fait d'ailleurs pas de mystère du rapport ambigu qui existe entre L'Imaginaire et l'œuvre husserlienne :

J'étais « husserlien » et devais le rester longtemps. [...] J'écrivis tout un livre (moins les derniers chapitres) sous son inspiration : L'Imaginaire. Contre lui, à dire vrai, mais tout autant qu'un disciple peut écrire contre son maitre ${ }^{5}$.

Les derniers chapitres de L'Imaginaire, en particulier la conclusion qui développe l'idée d'une conscience néantisante, permettent en effet de localiser le

\footnotetext{
${ }^{1}$ Ibid., pp. 150-151.

${ }^{2}$ V. de Coorebyter, « De Husserl à Sartre », art. cit., p. 3.

${ }^{3}$ Ibid., p. 3.

${ }^{4}$ Ibid., p. 4

${ }^{5}$ J.-P. Sartre, Carnets de la drôle de guerre, op. cit., p. 467.
} 
point de séparation entre les œuvres de Sartre et Husserl. Selon Jean Hyppolite, cette césure débute à partir de la catégorie de la négation. Pour lui, c'est "en interprétant l'intentionnalité comme une négation de l'être " ${ }^{1}$ que Sartre a pu s'émanciper définitivement de la voie d'analyse offerte par la phénoménologie husserlienne ${ }^{2}$, et, par là même, déplacer l'axe des problématiques couvertes par la phénoménologie. Car l'œuvre sartrienne s'oriente « vers une perspective existentielle, et peut-être même éthique ${ }^{3}$, tandis que le projet de Husserl poursuit la découverte d'une théorie pure à partir de la réduction phénoménologique.

Plus précisément le concept de négation représente un enjeu capital dans l'œuvre sartrienne, dans la mesure où il permet d'articuler les notions d'image et d'intention à un programme de recherches plus vaste, qui occupe Sartre jusqu'à L'Idiot de la famille : celui d'une philosophie de la liberté du sujet, mais aussi du développement de sa singularité au travers d'un processus de personnalisation. L'acte d'imagination consiste en effet en une thèse d'irréalisation qui assure, au sein de l'économie générale de la conscience, une fonction de persuasion et de libération du sujet; tandis que, en ce qui concerne la personnalisation du pour-soi, l'intentionnalité imaginative crée et maintient le circuit de l'ipséité de la conscience en face des difficultés opposées par la situation. À n'en pas douter, la conscience imageante occupe donc une importance de premier plan dans l'œuvre sartrienne. Du moins, l'on espère que nos analyses ont contribué à mettre en évidence l'enjeu intrinsèquement lié à la phénoménologie sartrienne de l'image : celui d'une philosophie consistante de la liberté personnelle du sujet en situation.

\footnotetext{
${ }^{1}$ J.-P. Hyppolite, Figures de la pensée philosophique, op. cit., p. 760.

${ }^{2}$ Très récemment, les Nouvelles lectures de L'Être et le Néant ont réitéré le constat de J. Hyppolite ( $C f$. J.-P. Narboux, «Intentionnalité et négation dans L'Être et le néant », dans Sartre. L'Être et le néant. Nouvelles lectures, op. cit., p. 57-91). En nous basant sur le volume vingt-trois des Husserliana, l'on pourrait toutefois nuancer cette lecture de Sartre. L'Imaginaire développe peut-être, en effet, une intuition déjà présente dans l'œuvre de Husserl. Dans ses cours à Göttingen, en 1904-1095, ce dernier soutient notamment que "l'objet-image est un néant» (E. Husserl, Phantasia, conscience d'image, souvenir. De la phénoménologie des présentifications intuitives. Textes posthumes, Husserliana XXIII, trad. fr. R. Kassis et J.-F. Pestureau, Grenoble, Millon, 2002, p. 456).

${ }^{3}$ J.-P. Hyppolite, Figures de la pensée philosophique, op. cit., p. 762.
}

\section{1}




\section{Bibliographie}

Breeur R., Autour de Sartre. La conscience mise à nu, Grenoble, Millon, 2005.

De Coorebyter V., « De Husserl à Sartre. La structure intentionnelle de l'image dans L'Imagination et L'Imaginaire », Méthodos $\mathrm{n}^{\circ} 12$ (2012).

De Coorebyter V., Sartre face à la phénoménologie, Bruxelles, Ousia, 2000.

Descartes R., Méditations métaphysiques, Paris, GF Flammarion, 2011.

Granel G., Traditionis traditio, Paris, Gallimard, 1972.

Heidegger M., Ce qui fait l'être-essentiel d'un fondement ou "raison », trad. fr. H. Corbin, dans Id., Questions I \& II, Paris, Gallimard, 1968.

Hervy A., "Perception et imagination: La problématique des actes mixtes », Bulletin d'analyse phénoménologique IX 1 (2013), p. 1-29.

Husserl E., Phantasia, conscience d'image, souvenir. De la phénoménologie des présentifications intuitives. Textes posthumes, Husserliana XXIII, trad. fr. R. Kassis et J.-F. Pestureau, Grenoble, Millon, 2002.

Husserl E., Recherches logiques, trad. fr. H. Elie et alii, Paris, PUF, 2003.

Hyppolite J., Figures de la pensée philosophique, Paris, PUF, 1972.

Kant I., Critique de la raison pure, trad. fr. A. J.-L. Delamarre et F. Marty, Paris, Gallimard, 1980.

Mouillie J.-M., Sartre : Conscience, Ego et Psyché, PUF, 2000.

Narboux J.-P., «Intentionnalité et négation dans L'Être et le néant», dans Sartre. L'Être et le néant. Nouvelles lectures, op. cit., p. 57-91.

Recchia F., «Émotion, ipséité, liberté : Réflexions à propos des fondements de la théorie sartrienne des émotions », Bulletin d'analyse phénoménologique XII 5 (2016), p. 1-23.

Ricœur P., À l'école de la phénoménologie, Paris, Vrin, 1998.

Rizk H., "Être et faire, la liberté comme principe d'individuation », dans J.M. Mouillie et J.-P. Narboux (dir.), Sartre. L'Être et le néant. Nouvelles lectures, Paris, Les Belles Lettres, 2015, p. 193-212.

Sartre J.-P., « Une idée fondamentale de la phénoménologie de Husserl : l'intentionnalité », dans Id., La Transcendance de l'Ego et autres textes phénoménologiques, Paris, Vrin, 2003, p. 87-89.

Sartre J.-P., Carnets de la drôle de guerre, dans Id., Les Mots et autres écrits autobiographiques, Paris, Gallimard, 2010.

Sartre J.-P., L'Être et le Néant, Paris, Gallimard, 1976.

Sartre J.-P., L'Idiot de la famille, Paris, Gallimard, 1988.

Sartre J.-P., L'Imaginaire, Paris, Gallimard, 2005.

Sartre J.-P., L'Imagination, Paris, PUF, 2010.

Sartre J.-P., La Transcendance de l'Ego, Paris, Vrin, 2012.

Sartre J.-P., Lettres au Castor et à quelques autres, Paris, Gallimard, 1983. 